\title{
Long-Term Prognosis and Predictive Risk Factors for Polyvascular Disease in Patients with Peripheral Arterial Disease
}

\author{
Kuniki Nakashima1, Hisao Kumakura2*, Ryuichi Funada², Yae Matsuo², \\ Kimimasa Sakata ${ }^{1}$, Akiko Ichikawa ${ }^{3}$, Toshiya Iwasaki ${ }^{2}$, Shuichi Ichikawa ${ }^{2}$
}

\author{
${ }^{1}$ Department of Cardiovascular Surgery, Cardiovascular Hospital of Central Japan (Kitakanto Cardiovascular Hospital), Gunma, \\ Japan \\ ${ }^{2}$ Department of Cardiovascular Medicine, Cardiovascular Hospital of Central Japan (Kitakanto Cardiovascular Hospital), Gunma, \\ Japan \\ ${ }^{3}$ Department of Nephrology, Cardiovascular Hospital of Central Japan (Kitakanto Cardiovascular Hospital), Gunma, Japan \\ Email: *kumakura@r.sannet.ne.jp
}

How to cite this paper: Nakashima, K. Kumakura, H., Funada, R., Matsuo, Y., Sakata, K., Ichikawa, A., Iwasaki, T. and Ichikawa, S. (2022) Long-Term Prognosis and Predictive Risk Factors for Polyvascular Disease in Patients with Peripheral Arterial Disease. World Journal of Cardiovascular Diseases, 12, 50-64.

https://doi.org/10.4236/wjcd.2022.121006

Received: December 1, 2021

Accepted: January 24, 2022

Published: January 27, 2022

Copyright $\odot 2022$ by author(s) and Scientific Research Publishing Inc. This work is licensed under the Creative Commons Attribution International License (CC BY 4.0).

http://creativecommons.org/licenses/by/4.0/

\begin{abstract}
Background: The aim of the current study was to assess fifteen-year life expectancy, cardiovascular events, fate of the limb, and risk factors with or without polyvascular disease in patients with Peripheral Arterial Disease (PAD). Methods: We conducted a prospective cohort study in 1019 PAD patients. The endpoints were Cardiovascular or Cerebrovascular Death (CCVD), All-Cause Death (ACD), Major Adverse Cardiovascular Events (MACE), and Cardiovascular and/or Limb Events (CVLE). Results: The patients who died were $539(52.9 \%)$ during follow-up periods. The rate of CCVD was $50.5 \%(\mathrm{n}=$ 272). In multiple regression analysis, the number of affected arteries had correlations with estimated Glomerular Filtration Rate (eGFR), HDL-cholesterol, Ankle Brachial Pressure Index (ABI), and diabetes $(\mathrm{p}<0.05)$. In multiple logistic analysis, PAD with Cerebrovascular Disease (CVD) was correlated with older age, ABI, eGFR, and atrial fibrillation ( $\mathrm{p}<0.05)$; PAD with Coronary Heart Disease (CHD) was correlated with younger age, eGFR, HDL-cholesterol, LDLcholesterol, and diabetes ( $\mathrm{p}<0.05$ ); and triple vascular disease (PAD with CVD and $\mathrm{CHD}$ ) was correlated with ABI, eGFR, HDL-cholesterol, and diabetes ( $\mathrm{p}$ $<0.05)$. The number of affected arteries had significant correlations with CCVD, ACD, MACE, and CVLE ( $\mathrm{p}$ < 0.05). In Cox multivariate analyses, age, Critical Limb Ischemia (CLI), eGFR, albumin, C-Reactive Protein (CRP), Body Mass Index (BMI), CVD, and CHD were related to CCVD ( $\mathrm{p}<0.05)$; age, albumin, eGFR, CRP, BMI, CLI, and CVD were associated with ACD ( $p<0.05)$; age, albumin, eGFR, CRP, CLI, CHD, and diabetes were associated with MACE (p $<0.05$ ); age, ABI, albumin, eGFR, CRP, CLI, CHD, and diabetes were related
\end{abstract}


to CVLE $(\mathrm{p}<0.05)$. Statins improved all outcomes $(\mathrm{p}<0.05)$. Conclusions: Polyvascular disease was independently associated with fifteen-year mortality, cardiovascular events, and the fate of the limb with diverse risk factors in PAD patients.

\section{Keywords}

Polyvascular Disease, Cerebral Infarction, Coronary Heart Disease, Fate of Leg, Peripheral Arterial Disease

\section{Introduction}

Polyvascular disease is defined as a coexistent arterial disease in two or three territories (cerebral, coronary, and/or peripheral) within each patient [1]. Patients with Peripheral Arterial Disease (PAD) complicate with severe systemic atherosclerosis that causes mortality due to Cerebrovascular Disease (CVD) and Coronary Heart Disease (CHD) [2] [3] [4] [5]. Since atherosclerosis is a systemic disease process, recent attention has focused on the coincidence of PAD with atherosclerosis in other arterial beds, most commonly CHD and CVD. In the Reduction of Atherothrombosis for Continued Health (REACH) Registry [6], the prevalence complicated with other arterial diseases was $22.4 \%$ in CHD (PAD and/or CVD) and $23.0 \%$ in CVD (PAD and/or CHD), whereas that was $43.8 \%$ in PAD (CVD and/or CHD) [7]. Moreover, several studies have reported that half of the patients with PAD have concomitant CHD [2] [8] [9]. We have found the prevalence of asymptomatic CVD is extremely high and CVD is an independent risk factor for long-term survival and aggravation of limb stages in PAD patients [10] [11]. Thus, patients with PAD have the most severe systemic atherosclerosis that causes higher morbidity and mortality among these three vascular diseases [12] [13]. In addition to the high mortality risk due to CHD and CVD, there is a higher probability of acute limb ischemia, revascularization, and amputation in patients with atherosclerosis of lower limb vessels among PAD patients with polyvascular disease [14].

Several studies have reported risk factors for concomitant vascular diseases in PAD patients including PAD alone (PADa), double vascular diseases (DoVD: PAD with CVD or CHD), and triple vascular diseases (TrVD: PAD with CVD and CHD) [1] [7] [15]. A number of risk factors for poorer outcomes have been identified in these patients, with polyvascular disease with Chronic Kidney Disease (CKD) and/or Diabetes Mellitus (DM) particularly markedly increasing the risk of cardiovascular events and/or heart failure [16] [17]. However, extremely long-term life expectancy, cardiovascular or cerebrovascular events, and the fate of the limb have not been confirmed in recent clinical follow-up data including causative risk factors in PAD patients. The purpose of the current study was to assess these outcomes for fifteen-year and causative risk factors with or without coexistent other vascular diseases in patients with PAD. 


\section{Patients and Methods}

\subsection{Patients}

The subjects included Japanese patients with PAD who were hospitalized in Cardiovascular Hospital of Central Japan during the period from February 1, 2000 and April 30, 2020. This prospective cohort study complies with the principles of the Declaration of Helsinki, and the Medical Ethical Committee approved the study protocol in our hospital (CCJ-EA-006). Patients who gave their written informed consent to partake in this study were chosen as subjects. Patients satisfying the following inclusion criteria were selected: 1) an Ankle Brachial Pressure Index (ABI) of $<0.90 ; 2$ ) clinical symptoms (intermittent claudication or critical limb ischemia); 3) a stenotic lesion of $\geq 70 \%$ in iliac or femoropopliteal artery was specified with angiography or ultrasound. Patients with a treatment history of PAD including amputation of leg in another side were excluded from this study. The patients were also limited to those with PAD due to atherosclerosis. Patients with PAD due to non-atherosclerotic causes, such as vasculitis, Buerger disease, and fibromuscular dysplasia, were excluded. Patients with dementia were also excluded due to the difficulty of checking vital signs and obtaining other information.

\subsection{Baseline Clinical Characteristics}

Clinical data characteristics for each patient were obtained from the primary analysis for age, Body Mass Index (BMI), ABI, smoking history, hypertension, and Diabetes Mellitus (DM). The concentration of creatinine, albumin, triglyceride, total cholesterol, High Density Lipoprotein Cholesterol (HDL-C), Low Density Lipoprotein Cholesterol (LDL-C), glucose, D-dimer, and C-reactive protein (CRP) was determined with Hitachi 7180 automatic analyzer (Hitachi High-Tech Fielding Co., Tokyo, Japan). DM was defined as a fasting glucose level of $>126 \mathrm{mg} / \mathrm{dL}$ at least two data or receiving antidiabetic treatment [18]. Hypertension was specified as blood pressure $\geq 140 / 90 \mathrm{mmHg}$ recorded at least twice or a requirement for antihypertensive treatment. The estimated Glomerular Filtration Rate (eGFR) was calculated using the Modification of Diet in Renal Disease equation with age and serum creatinine level [19].

\subsection{Assessment of CVD and CHD}

A brain Computed Tomography (CT) scan was performed in 5- or 10-mm thick sections without contrast agent (Aquilion 64, Toshiba, Tokyo, Japan). The scans were evaluated by two radiologists who were blinded to the statuses of the patients. Infarcts $>1.5 \mathrm{~cm}$ in diameter were defined to be cerebral infarction on brain CT. A low density lesion with a diameter $\leq 1.5 \mathrm{~cm}$ was defined as a lacunar infarction. Patients with CVD were defined as cerebral infarction and/or lacunar infarction on brain CT or a history of this disease. An electrocardiogram and an echocardiography were performed for each patient. CHD was defined as a documented history of ischemic heart disease (myocardial infarction, percutaneous 
coronary intervention, composite of angina pectoris, or coronary artery bypass grafting) or a coronary angiogram ( $\geq 50 \%$ stenosis in at least one coronary artery).

\subsection{Data Analysis and Endpoints}

Each patient was followed up at 1, 3 and 4 months after treatment and assessed interval was 4- or 6-month. Vital signs and medical status were assessed with hospital data and written questionnaires for life statuses were assessed by the Foot Care Club [4] [20]. Ischemic strokes were determined as the presence of a new focal neurological deficit, and magnetic resonance imaging or CT was required to confirm the lesions. TIA was specified as the presence of a new neurological deficit lasting $<24$ hours. Definition of myocardial infarction was signified previously [20] [21]. Peripheral restenosis during follow-up was determined as a decrease in $\mathrm{ABI}$ of $\geq 0.15 \%$ and $\geq 50 \%$ stenosis using angiography or duplex ultrasonography [20] [22], and major amputation was defined as above-the-ankle amputation.

The primary efficacy endpoints were Cardiovascular or Cerebrovascular Related Death (CCVD) and All-Cause Death (ACD). The secondary efficacy end-points were major adverse cardiovascular events (MACE: all-cause death, non-fatal myocardial infarction, non-fatal ischemic stroke, or transient ischemic attack) and cardiovascular and/or limb events (CVLE: CCVD, non-fatal myocardial infarction or cerebral infarction, transient ischemic attack, presence of a new peripheral lesion, repeat revascularization for a limb, or major amputation).

\subsection{Statistical Analysis}

All statistical analyses were calculated with IBM SPSS Statistics ver. 25.0 (IBM Corp, Armonk, NY). Categorical variables are signified as a number (\%) and were compared by chi-square test with a Bonferroni correction. Continuous variables are expressed as a median (interquartile range) and were assessed by Kruskal-Wallis test with the Dann-Bonferroni method [23]. In multiple regression analysis, we calculated among all risk factors with simple Pearson correlations. Factors with $\mathrm{p}<0.05$ in this correlation analysis were calculated using stepwise forward multiple regression analysis to define relationships between the number of affected arteries and individual risk factors. We calculated Odds Ratio (OR) and Confidence Interval (CI) between DoVD or TrVD and risk factors with a univariate logistic analysis. Factors with $\mathrm{p}<0.05$ in these analyses were used in a multivariate logistic model to define the risk predictors for DoVD and TrVD. The Kaplan-Meier analysis was used to specify CCVD, ACD, MACE, and CVLE among PADa, DoVD, or TrVD and compared using the log-rank test with Bonferroni correction. In Cox univariate regression model, a Hazard Ratio (HR) and 95\% CI were estimated for individual factors. Factors with $\mathrm{p}<0.05$ were used in multivariate regression models to define significant factors associated with these endpoints. A p-value < 0.05 was defined as statistically significant. 


\section{Results}

\subsection{Patient Characteristics and Causes of Death}

Among 1053 patients, follow-up was possible for 1019 subjects. The mean and median follow-up periods were $80.2 \pm 65.9$ and 67 (29 - 115) months. The median and mean age were $72(67-79)$ and $72.6 \pm 9.9$ years. The patients who died were 539 (52.9\%) during the follow-up periods. The prevalence of CCVD was 50.5\% $(\mathrm{n}=272)$, as cardiac or major vascular disease $(\mathrm{n}=193,35.8 \%)$, cerebrovascular disease $(n=79,14.7 \%)$. Other causes of deaths were malignancy $(n=108,20.0 \%)$, pneumonia $(\mathrm{n}=88,16.3 \%)$, and other causes $(\mathrm{n}=71,13.2 \%)$. The cumulative 5-, 10-, and 15-year overall survival rates in all patients were $71.8 \%, 45.4 \%$, and $32.7 \%$, respectively. The baseline clinical characteristics and comorbidities in PAD patients with or without other vascular diseases are summarized in Table 1. Patients with PADa had higher ABI, serum albumin, eGFR, and HDL-C, and lower levels of CRP and D-dimer. The prevalences of CLI and DM were also lower in these patients.

\subsection{Number of Affected arteries and Risk Factors}

The number of affected arteries had significant positive correlations with age, men, CLI, LDL-C, and DM, and negative correlations with ABI, HDL-C, serum albumin, and eGFR in simple Pearson correlation analysis $(\mathrm{p}<0.05)$. Stepwise forward multiple regression analysis of the relationship between the number of affected arteries and these factors was performed (Table 2). The number of affected arteries had significant negative correlations with eGFR, HDL-C, and ABI, and a positive correlation with DM $(\mathrm{p}<0.01)$.

\subsection{Risk Predictors for DoVD and TrVD}

Relationships between risk factors and DoVD and TrVD were analyzed using multiple logistic analyses (Table 3). PAD with CVD was correlated with older age, lower ABI, eGFR, and atrial fibrillation; PAD with CHD showed a correlation with younger age, lower eGFR, HDL-C, higher LDL-C, and DM; and TrVD was correlated with lower $\mathrm{ABI}$, eGFR, HDL-C, and DM.

\subsection{Factors for ACD and CCVD}

Cumulative incidence of the 5-, 10-, and 15-year rates for CCVD are demonstrated in Figure 1. There were significant differences among PADa, DoVD, or TrVD ( $\mathrm{p}$ $<0.001$, respectively). In Cox univariate analysis, age, CLI, CVD, CHD, hypertension, higher level of CRP, D-dimer, lower ABI, BMI, serum albumin, eGFR, and statin were associated to CCVD ( $<0.05)$. In multivariate analysis, higher age, CRP, lower serum albumin, eGFR, BMI, CLI, CVD, and CHD were also associated with CCVD, and statin also decreased CCVD (Table 4, $\mathrm{p}<0.05$ ).

The cumulative 5-, 10-, and 15-year incidence rates for ACD are demonstrated in Figure 2. There were significant differences between PADa and DoVD (p $<0.001)$ or $\operatorname{TrVD}(\mathrm{p}<0.001)$, but there was no significant difference between DoVD 
and $\operatorname{TrVD}(\mathrm{p}=0.066)$. In Cox univariate analysis, age, CLI, CVD, DM, higher level of CRP, D-dimer, lower ABI, BMI, serum albumin, and eGFR were associated with $\mathrm{ACD}(\mathrm{p}<0.05)$. Treatment with statin or aspirin and revascularization were related to ACD. In multivariate analysis, higher age, CLI, CRP, lower BMI, serum albumin, eGFR, and CVD were also associated with ACD, and statin decreased ACD (Table 4, p < 0.05).

\subsection{Factors for MACE and CVLE}

The cumulative 5-, 10-, and 15-year incidence rates for MACE are shown in.

Table 1. Clinical characteristics and comorbidities in patients with Peripheral Arterial Disease (PAD) with or without other Vascular Disease (VD).

\begin{tabular}{|c|c|c|c|}
\hline Factor & $\begin{array}{c}\text { PAD alone } \\
\mathrm{n}=256(25.1 \%)\end{array}$ & $\begin{array}{c}\text { Double VD } \\
\mathrm{n}=540(53.0 \%)\end{array}$ & $\begin{array}{c}\text { Triple VD } \\
\mathrm{n}=223(21.9 \%)\end{array}$ \\
\hline Age (year) & $73(65-79)$ & $74(67-80)$ & $73(67-79)$ \\
\hline Gender (men) & $177(69.1 \%)$ & $418(77.4 \%)^{\star}$ & $182(81.6 \%)^{\S}$ \\
\hline Ankle brachial pressure index & $0.73(0.56-0.88)$ & $0.66(0.51-0.80)^{\star}$ & $0.67(0.50-0.80)^{\S}$ \\
\hline Body mass index $\left(\mathrm{kg} / \mathrm{m}^{2}\right)$ & $22.8(20.0-25.1)$ & $22.0(20.0-24.2)$ & $22.2(20.0-24.3)$ \\
\hline Critical limb ischemia & $29(11.3 \%)$ & $106(19.6 \%)^{\star}$ & $44(19.7 \%)^{\S}$ \\
\hline Intermittent claudication & $227(88.7 \%)$ & $434(80.4 \%)^{\star}$ & $179(80.3 \%)^{\S}$ \\
\hline Coronary heart disease & 0 & $217(40.2)$ & $223(100 \%)$ \\
\hline Cerebral infarction & 0 & $323(59.8)$ & $223(100 \%)^{9}$ \\
\hline Diabetes mellitus & $85(33.2 \%)$ & $208(38.5 \%)^{\star}$ & $119(53.4 \%)^{59}$ \\
\hline Hypertension & $161(62.9 \%)$ & $372(68.9 \%)$ & $154(69.1 \%)$ \\
\hline Smoking & $178(69.8 \%)$ & $407(75.4 \%)$ & $163(73.1 \%)$ \\
\hline Hemodialysis & $17(6.6 \%)$ & $54(10.0 \%)^{\star}$ & $40(17.9 \%)^{\S 9}$ \\
\hline Atrial fibrillation & $16(6.3 \%)$ & $70(13.0 \%)^{\star}$ & $23(10.3 \%)$ \\
\hline \multicolumn{4}{|l|}{ Basic metabolic panel } \\
\hline eGFR $\left(\mathrm{mL} / \mathrm{min} / 1.73 \mathrm{~m}^{2}\right)$ & $56.6(51.9-73.2)$ & $56.8(42.5-69.4)^{\star}$ & $50.6(31.9-66.7)^{\S}$ \\
\hline Albumin (g/dL) & $4.1(3.8-4.2)$ & $4.0(3.7-4.2)^{\star}$ & $3.9(3.7-4.2)^{\mathfrak{S}}$ \\
\hline D-dimer $(\mu \mathrm{g} / \mathrm{dL})$ & $0.7(0.5-1.4)$ & $1.0(0.5-2.2)^{\star}$ & $1.1(0.6-2.2)^{\S}$ \\
\hline C-reactive protein $(\mathrm{mg} / \mathrm{dL})$ & $0.14(0.07-0.32)$ & $0.18(0.08-0.50)^{\star}$ & $0.21(0.10-0.54)^{\S}$ \\
\hline Total cholesterol (mg/dL) & $181(152-214)$ & $189(160-215)$ & $193(171-223)^{\S}$ \\
\hline Triglyceride (mg/dL) & $125(89-173)$ & $129(87-203)$ & $128(95-179)$ \\
\hline HDL-C (mg/dL) & $52(42-62)$ & $47(40-58)^{\star}$ & $46(36-54)^{\S}$ \\
\hline LDL-C (mg/dL) & $111(90-128)$ & $114(90-135)$ & $117(97-137)$ \\
\hline \multicolumn{4}{|l|}{ Medications } \\
\hline Thienopyridines & $102(39.8 \%)$ & $276(51.1 \%)^{\star}$ & $135(60.5 \%)^{\varsigma s}$ \\
\hline Aspirin & $168(65.6 \%)$ & $393(72.9 \%)$ & $184(82.5 \%)^{\S}$ \\
\hline Beraprost & $103(40.2 \%)$ & $205(38.0 \%)$ & $70(31.4 \%)$ \\
\hline Cilostazol & $60(23.4 \%)$ & $162(30.0 \%)$ & $48(21.5 \%)^{9}$ \\
\hline
\end{tabular}




\section{Continued}

Ca antagonist

ACE inhibitor

ARB

$\beta$-blocker

Statin

Revascularization

$$
\begin{gathered}
134(52.3 \%) \\
26(10.2 \%) \\
65(25.4 \%) \\
24(9.4 \%) \\
156(60.9 \%)
\end{gathered}
$$$$
143 \text { (55.9\%) }
$$

289 (53.5\%)

$115(51.6 \%)$

$56(10.4 \%)$

165 (30.6\%)

88 (16.3\%)

$339(62.8 \%)$

$324(60.0 \%)$
$26(11.7 \%)$

$85(38.1 \%)^{\S}$

$50(22.4 \%)^{\text {sg }}$

$153(68.6 \%)$

143 (64.4\%)

Double VD: PAD with cerebral infarction or coronary heart disease; Triple VD: PAD with cerebral infarction and coronary heart disease; eGFR: estimated glomerular filtration rate; LDL-C: low density lipoprotein cholesterol; HDL-C: high density lipoprotein cholesterol; ACE: angiotensin-converting enzyme; ARB: angiotensin receptor blocker. ${ }^{\star}: \mathrm{p}<0.05$ (PAD alone vs. Double VD); ${ }^{\varsigma}: \mathrm{p}<0.05$ (PAD alone vs. Triple VD); ${ }^{\text {: }} \mathrm{p}<0.05$ (Double VD vs. Triple VD).

Table 2. Correlations between the number of affected arteries and other risk factors in stepwise forward multiple regression analysis.

\begin{tabular}{|c|c|c|c|c|c|c|c|c|c|}
\hline \multirow{3}{*}{ Risk factor } & \multicolumn{6}{|c|}{ Double vascular diseases } & \multirow{2}{*}{\multicolumn{3}{|c|}{$\begin{array}{c}\text { Triple vascular diseases } \\
\mathrm{PAD}, \mathrm{CVD} \text {, and } \mathrm{CHD}(\mathrm{n}=223)\end{array}$}} \\
\hline & \multicolumn{3}{|c|}{ PAD and CVD $(n=323)$} & \multicolumn{3}{|c|}{ PAD and CHD $(n=217)$} & & & \\
\hline & OR & $95 \% \mathrm{CI}$ & p-value & OR & $95 \% \mathrm{CI}$ & p-value & OR & $95 \% \mathrm{CI}$ & p-value \\
\hline Age (year) & 1.018 & $1.001-1.037$ & 0.048 & 0.976 & $0.955-0.998$ & 0.030 & & & \\
\hline $\mathrm{ABI}$ & 0.353 & $0.181-0.691$ & 0.002 & & & & 0.419 & $0.184-0.953$ & 0.038 \\
\hline Diabetes mellitus & & & & 1.598 & $1.040-2.456$ & 0.032 & 2.332 & $1.516-3.626$ & $<0.001$ \\
\hline $\mathrm{eGFR}\left(\mathrm{mL} / \mathrm{min} / 1.73 \mathrm{~m}^{2}\right)$ & 0.992 & $0.986-0.990$ & 0.007 & 0.990 & $0.980-0.999$ & 0.034 & 0.982 & $0.972-0.992$ & $<0.001$ \\
\hline HDL-C (mg/dL) & & & & 0.978 & $0.964-0.993$ & 0.004 & 0.979 & $0.964-0.994$ & 0.005 \\
\hline LDL-C (mg/dL) & & & & 1.005 & $1.001-1.010$ & 0.017 & & & \\
\hline Atrial fibrillation & 1.973 & $1.046-3.724$ & 0.036 & & & & & & \\
\hline
\end{tabular}

\begin{tabular}{ccccc}
\hline Risk factor & $\beta$ & B & 95\% C.I. & P-value \\
\hline eGFR $\left(\mathrm{mL} / \mathrm{min} / 1.73 \mathrm{~m}^{2}\right)$ & -0.134 & -0.004 & -0.006 to -0.002 & $<0.001$ \\
Diabetes mellitus & 0.127 & 0.176 & 0.083 to 0.270 & 0.001 \\
HDL-C $(\mathrm{mg} / \mathrm{dL})$ & -0.115 & -0.005 & -0.008 to -0.002 & 0.001 \\
ABI & -0.104 & -0.255 & -0.418 to -0.093 & 0.002
\end{tabular}

$\mathrm{R}^{2}=0.059 ; \mathrm{F}$ for change in $\mathrm{R}^{2}=0.009 ; \mathrm{P}=0.006 . \beta$ : standardized coefficient; $\mathrm{B}$ : non-standardized coefficient; CI: confidence interval for B; eGFR: estimated glomerular filtration rate; ABI: ankle brachial pressure index.

Table 3. Relationships between three arterial lesion levels and risk factors in multiple logistic analysis.

PAD: peripheral arterial disease; CVD: cerebrovascular disease; CHD: coronary heart disease; OR: odds ratio; CI: confidence interval; ABI: ankle brachial pressure index; eGFR: estimated glomerular filtration rate; HDL-C: high density lipoprotein cholesterol; LDL-C: low density lipoprotein cholesterol. 


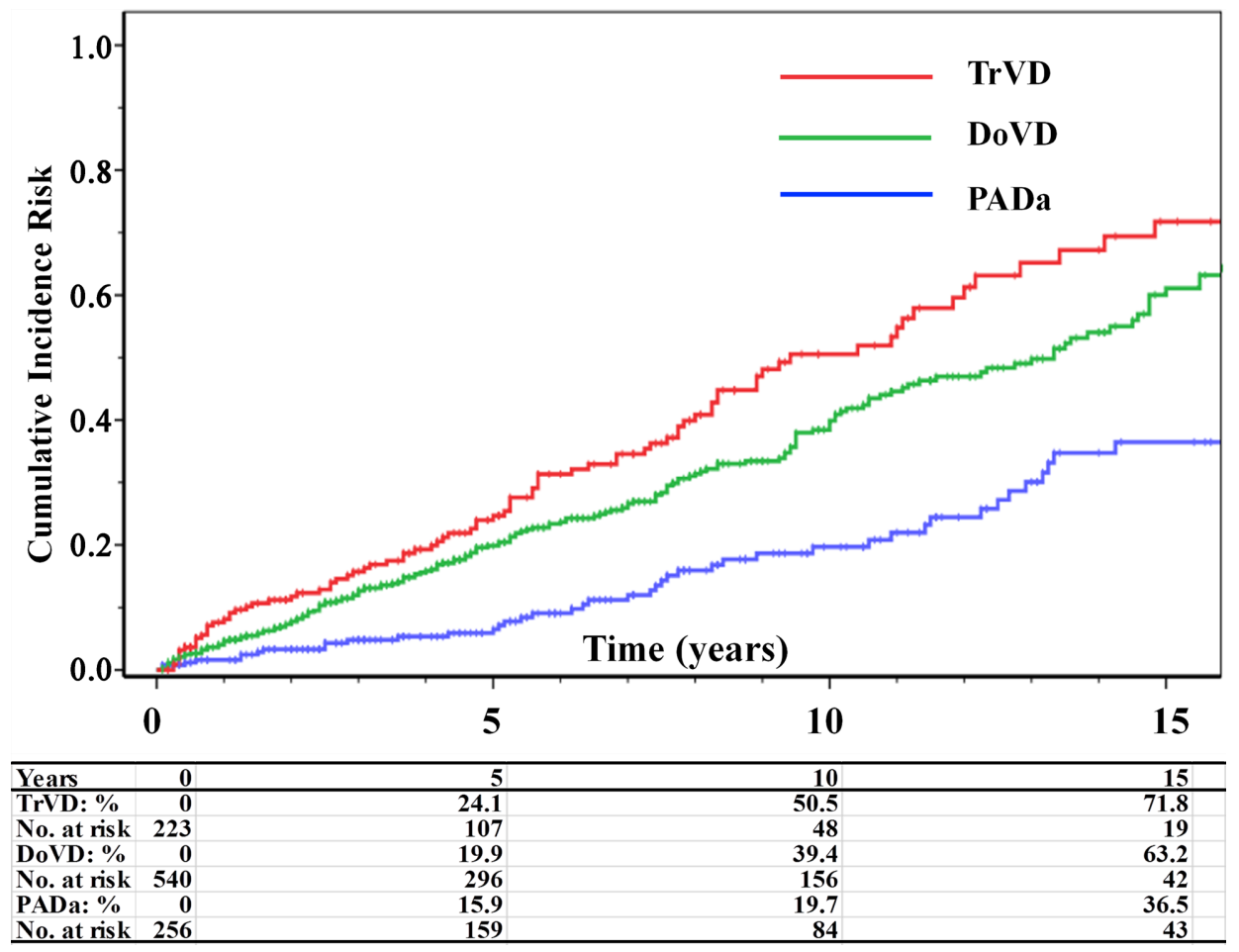

Figure 1. Cumulative 5-, 10-, and 15-year incidence rates for cardiovascular or cerebrovascular related death (CCVD) are demonstrated with significant differences among PAD alone (PADa), double vascular diseases (DoVD: PAD with CVD or CHD), or triple vascular diseases (TrVD: PAD with CVD and CHD) (all $\mathrm{p}<0.001)$.

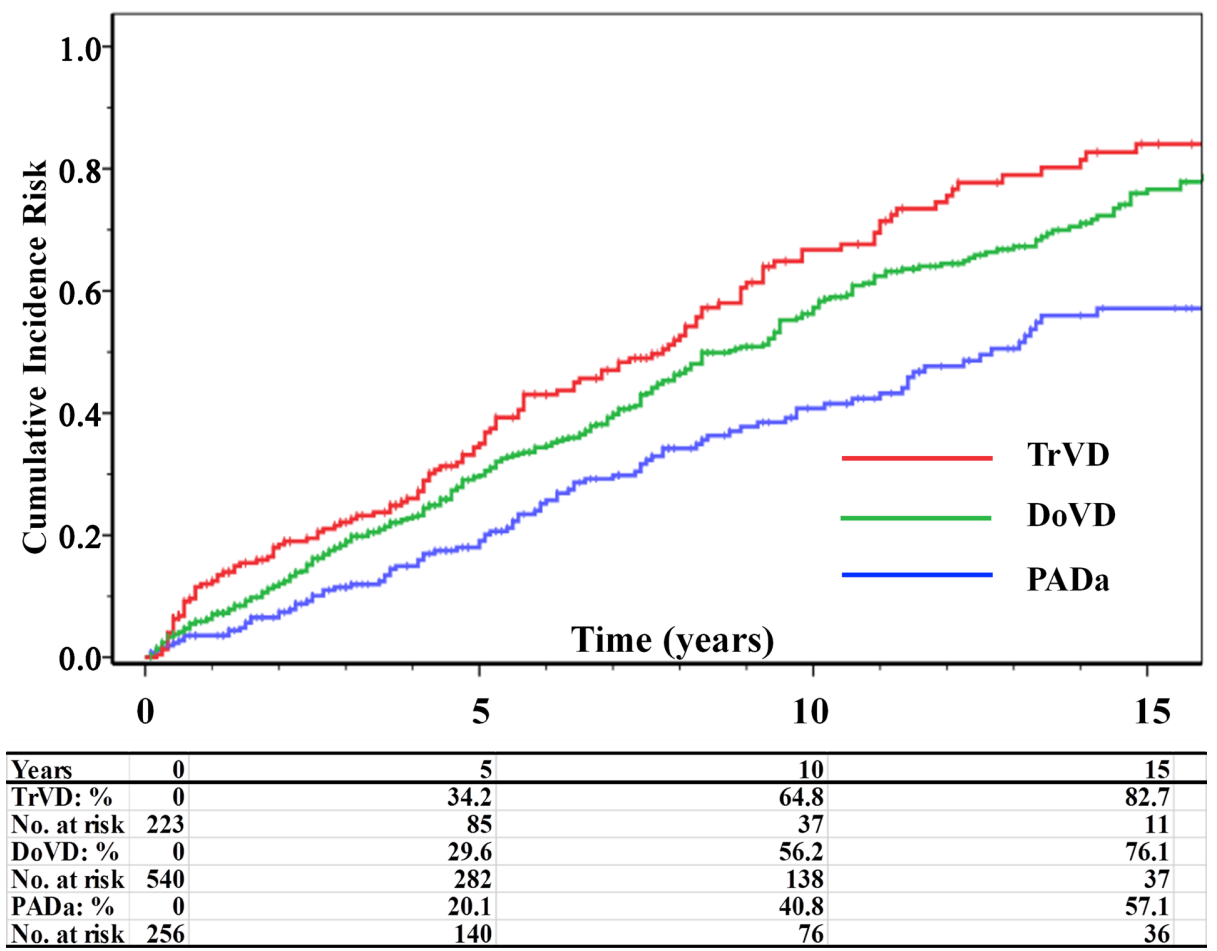

Figure 2. Cumulative 5-, 10-, and 15-year incidence rates for all-cause death (ACD) in PAD alone (PADa), double vascular diseases (DoVD: PAD with CVD or CHD) or triple vascular diseases (TrVD: PAD with CVD and CHD). There were significant differences between PADa and DoVD $(p<0.001)$ or TrVD $(p<0.001)$, but there was no significant difference between DoVD and $\operatorname{TrVD}(\mathrm{p}=0.066)$. 
Table 4. Cox multivariate regression analysis for Cardiovascular or Cerebrovascular Related Death (CCVD) and All-Cause Death (ACD).

\begin{tabular}{ccccccc}
\hline & \multicolumn{3}{c}{ CCVD } & \multicolumn{3}{c}{ ACD } \\
\cline { 2 - 7 } Factor & \multicolumn{3}{c}{ Multivariate analysis } & \multicolumn{3}{c}{ Multivariate analysis } \\
\cline { 2 - 7 } & HR & $95 \%$ CI & p-value & HR & 95\% CI & p-value \\
\hline Age (year) & 1.051 & $1.036-1.067$ & $<0.001$ & 1.054 & $1.042-1.066$ & $<0.001$ \\
Body mass index $\left(\mathrm{kg} / \mathrm{m}^{2}\right)$ & 0.955 & $0.916-0.996$ & 0.030 & 0.949 & $0.919-0.981$ & 0.002 \\
Critical limb ischemia & 1.939 & $1.375-2.732$ & $<0.001$ & 1.609 & $1.223-2.117$ & 0.001 \\
Cerebral infarction & 1.666 & $1.230-2.255$ & 0.001 & 1.338 & $1.068-1.676$ & 0.011 \\
Coronary heart disease & 1.477 & $1.119-1.949$ & 0.006 & 1.184 & $0.951-1.474$ & 0.131 \\
Serum albumin $(\mathrm{g} / \mathrm{dL})$ & 0.587 & $0.418-0.823$ & 0.002 & 0.521 & $0.400-0.678$ & $<0.001$ \\
eGFR (mL/min/1.73 m $\left.{ }^{2}\right)$ & 0.984 & $0.978-0.991$ & $<0.001$ & 0.987 & $0.982-0.992$ & $<0.001$ \\
C-reactive protein $(\mathrm{mg} / \mathrm{dL})$ & 1.110 & $1.016-1.213$ & 0.021 & 1.128 & $1.055-1.207$ & $<0.001$ \\
Statin & 0.487 & $0.355-0.667$ & $<0.001$ & 0.469 & $0.368-0.598$ & $<0.001$ \\
\hline
\end{tabular}

HR: hazard ratio; CI: confidence interval; eGFR: estimated glomerular filtration rate.

Figure 3. There were significant differences among PADa, DoVD, or $\operatorname{TrVD}(\mathrm{p}<$ 0.001, respectively). In Cox univariate analysis, age, men, CLI, CVD, CHD, DM, higher level of CRP, D-dimer, lower BMI, ABI, serum albumin, eGFR, and statin were associated to MACE $(\mathrm{p}<0.05)$. In multivariate analysis, higher age, CRP, lower serum albumin, eGFR, CLI, CHD, and DM were also associated with MACE, and statin decreased MACE (Table 5, $\mathrm{p}<0.05$ ).

The cumulative 5-, 10-, and 15-year incidence rates for CVLE are shown in Figure 4. There were significant differences among PADa, DoVD, or TrVD



Figure 3. Cumulative 5-, 10-, and 15-year incidence rates for major adverse cardiovascular events (MACE) with significant differences among PAD alone (PADa), double vascular diseases (DoVD: PAD with CVD or CHD), or triple vascular diseases (TrVD: PAD with CVD and CHD) (all $\mathrm{p}<0.001$ ). 




Figure 4. Cumulative 5-, 10-, and 15-year incidence rates for cardiovascular and/or limb events (CVLE) with significant differences among PAD alone (PADa), double vascular diseases (DoVD: PAD with CVD or CHD), or triple vascular diseases (TrVD: PAD with CVD and CHD) (all $\mathrm{p}<0.001)$.

Table 5. Cox multivariate regression analysis for Major Adverse Cardiovascular Events (MACE) and Cardiovascular and/or Limb Events (CVLE).

\begin{tabular}{|c|c|c|c|c|c|c|}
\hline \multirow{3}{*}{ Factor } & \multicolumn{3}{|c|}{ MACE } & \multicolumn{3}{|c|}{ CVLE } \\
\hline & \multicolumn{3}{|c|}{ Multivariate analysis } & \multicolumn{3}{|c|}{ Multivariate analysis } \\
\hline & HR & $95 \% \mathrm{CI}$ & p-value & HR & $95 \% \mathrm{CI}$ & $\mathrm{p}$-value \\
\hline Age (year) & 1.038 & $1.028-1.049$ & $<0.001$ & 1.022 & $1.012-1.032$ & $<0.001$ \\
\hline $\mathrm{ABI}$ & 0.705 & $0.491-1.014$ & 0.059 & 0.663 & $0.469-0.936$ & 0.020 \\
\hline Critical limb ischemia & 1.447 & $1.125-1.862$ & 0.004 & 1.313 & $1.012-1.702$ & 0.040 \\
\hline Coronary heart disease & 1.841 & $1.514-2.238$ & $<0.001$ & 1.510 & $1.245-1.830$ & $<0.001$ \\
\hline Diabetes mellitus & 1.328 & $1.091-1.617$ & 0.005 & 1.331 & $1.066-1.672$ & 0.012 \\
\hline Serum albumin $(\mathrm{g} / \mathrm{dL})$ & 0.655 & $0.519-0.826$ & $<0.001$ & 0.717 & $0.571-0.900$ & 0.004 \\
\hline $\mathrm{eGFR}\left(\mathrm{mL} / \mathrm{min} / 1.73 \mathrm{~m}^{2}\right)$ & 0.994 & $0.990-0.999$ & 0.011 & 0.993 & $0.989-0.997$ & 0.001 \\
\hline C-reactive protein $(\mathrm{mg} / \mathrm{dL})$ & 1.114 & $1.048-1.185$ & 0.001 & 1.070 & $1.005-1.139$ & 0.035 \\
\hline Statin & 0.440 & $0.356-0.543$ & $<0.001$ & 0.474 & $0.387-0.580$ & $<0.001$ \\
\hline
\end{tabular}

HR: hazard ratio, CI: confidence interval, ABI: ankle brachial pressure index, eGFR: estimated glomerular filtration rate.

( $\mathrm{p}<0.001$, respectively). In Cox univariate analysis, age, men, CLI, CVD, CHD, DM, higher level of CRP, D-dimer, lower BMI, ABI, serum albumin, eGFR, and statin were associated to CVLE $(\mathrm{p}<0.05)$. In multivariate analysis, higher age, CRP, lower ABI, serum albumin, eGFR, CLI, CHD, and DM were also associated with CVLE, and statin also decreased CVLE (Table 5, $\mathrm{p}<0.05$ ). 


\section{Discussion}

This study assessed the first clinical evidence for fifteen-year mortality, cardiovascular or cerebrovascular events, and fate of the limb in PAD patients with or without polyvascular disease. When compared with PADa, patients with DoVD or TrVD had significantly higher rates of CCVD and ACD during the follow-up period. We found that the number of affected arteries had significant correlations with CCVD, MACE, and CVLE, and the risk of cardiovascular events increased in a stepwise manner with each additional arterial bed. Furthermore, lower eGFR, $\mathrm{ABI}$, and DM showed close relationships with the number of affected vascular territories and increased severity of vascular atherosclerosis.

The definition of coinstantaneous lesions of atherosclerotic disease in different vascular territories depends on the methods and criteria used for diagnosis. In particular, the criteria and definition for CVD and CHD are particularly dominan for diagnosis of DoVD or TrVD in polyvascular disease, and there is a risk of under diagnosis. Thus, we used severe threshold levels in the definitions for CVD and CHD in this study. Multiple comparisons among the three groups were difficult, but these analyses were instrumental in identifying risk factors and outcomes of polyvascular disease in patients with PAD.

We found several important differences between DoVD and TrVD. Within DoVD, there were some differences in the risk factor between CVD and CHD in multiple logistic analysis. The patient age as a risk factor was older in CVD and younger in CHD. In REACH Registry [6], the mean ages in these three arterial diseases were youngest in CHD, middle in CVD, and oldest in PAD. These basic characteristics may affect the differences of overlap between CVD and CHD in patients with PAD. Atrial fibrillation was an independent predictor for CVD. The patients with atrial fibrillation have an average annual risk of CVD of approximately 5\% [24]. Whereas, CHD was correlated with lower HDL-C, higher LDL-C, and DM. CHD is closely related lipid abnormality [25].

The prevalence of CCVD was $50.5 \%$ in ACD. CCVD was also strongly correlated with higher age, CRP, lower serum albumin, eGFR, BMI, CLI, CVD, and CHD. Together with higher age and CRP, DM is considered one of the strongest risk factors for PAD, CVD, and CHD [2] [11] [26]. Of importance, DM is particularly strongly associated with the severe stage in PAD as CLI [2] [26]. Furthermore, CVD was also an independent predictor for ACD. Severe systemic atherosclerosis reflected by CVD is responsible for ACD or CCVD [11].

Lower eGFR was an independent risk factor throughout DoVD and TrVD. Several studies have documented that lower eGFR is a prognostic indicator of CCVD [27] [28]. Moreover, we have also demonstrated lower BMI and geriatric nutritional risk index are significant predictive factors for ACD, CCVD, and CVLE in patients with PAD [4] [29]. The incidence of MACE or CVLE significantly increased with the number of arterial beds. Especially, CLI, lower eGFR, serum albumin, and higher CRP were significant risk factors for all outcomes. CLI and lower $\mathrm{ABI}$ are related to a higher risk of MACE and CVLE based on severe systemic 
atherosclerosis [4] [30]. These results documented that patient with chronic kidney disease, malnutrition, or severe PAD have systemic atherosclerosis as polyvascular disease which is the cause of morbidity or mortality.

Higher LDL-C had a significant correlation with CHD, and lower HDL-C were significantly associated with CHD and TrVD. Lipid abnormality is an independent risk factor for patients with polyvascular disease, and intensive lipidlowering therapy is effective for decreasing vascular events in these patients [1] [31]. Statins improve cardiovascular events and have an antiatherogenic effect on CHD [32]. Statin therapy is also effective for decreasing ACD and MACE in symptomatic and asymptomatic patients with PAD [33] [34]. These results suggest that statins also improve the long-term clinical risks for CCVD and CVLE.

\section{Limitations of the Study}

There are several limitations that should be considered in his study. First, the number of patients recruited to this study was relatively small. Second, the study was based on the data from a single facility. Third, the prescription rate of statins was relatively lower comparing to recent guidelines at the time of treatment, but the prevalence has increased over time. These results require further prospective long-term clinical follow-up data for these outcomes and risk factors in a larger cohort with PAD.

\section{Conclusion}

Polyvascular disease was independently associated with increased fifteen-year mortality, cardiovascular events, and the fate of the limb with diverse risk factors in PAD patients. The number of affected arteries had significant correlations with CCVD, ACD, MACE, and CVLE in patients with PAD.

\section{Acknowledgements}

We thank the vascular laboratory and clinical research staff in our hospital.

\section{Conflicts of Interest}

The authors declare no conflicts of interest.

\section{References}

[1] Poredos, P., Blinc, A., Novo, S. and Antignani, P.L. (2021) How to Manage Patients with Polyvascular Atherosclerotic Disease. Position Paper of the International Union of Angiology. International Angiology, 40, 29-41. https://doi.org/10.23736/S0392-9590.20.04518-6

[2] Norgren, L., Hiatt, W.R., Dormandy, J.A., Nehler, M.R., Harris, K.A. and Fowkes, F.G. (2007) Inter-Society Consensus for the Management of Peripheral Arterial Disease (TASC II). Journal of Vascular Surgery, 45, S5-S67. https://doi.org/10.1016/j.jvs.2006.12.037

[3] Banerjee, A., Fowkes, F.G. and Rothwell, P.M. (2010) Associations between Periph- 
eral Artery Disease and Ischemic Stroke: Implications for Primary and Secondary Prevention. Stroke, 41, 2102-2107. https://doi.org/10.1161/STROKEAHA.110.582627

[4] Kumakura, H., Kanai, H., Aizaki, M., Mitsui, K., Araki, Y., Kasama, S., et al. (2010) The Influence of the Obesity Paradox and Chronic Kidney Disease on Long-Term Survival in a Japanese Cohort with Peripheral Arterial Disease. Journal of Vascular Surgery, 52, 110-117. https://doi.org/10.1016/j.jvs.2010.02.008

[5] Murphy, T.P., Dhangana, R., Pencina, M.J. and D’Agostino, R.B.S. (2012) AnkleBrachial Index and Cardiovascular Risk Prediction: An Analysis of 11,594 Individuals with 10-Year Follow-Up. Atherosclerosis, 220, 160-167.

https://doi.org/10.1016/j.atherosclerosis.2011.10.037

[6] Ohman, E.M., Bhatt, D.L., Steg, P.G., Goto, S., Hirsch, A.T., Liau, C.S., et al. (2006) The REduction of Atherothrombosis for Continued Health (REACH) Registry: An International, Prospective, Observational Investigation in Subjects at Risk for Atherothrombotic Events-Study Design. American Heart Journal, 151, 786.e1-786.e10.

https://doi.org/10.1016/j.ahj.2005.11.004

[7] Yamazaki, T., Goto, S., Shigematsu, H., Shimada, K., Uchiyama, S., Nagai, R., et al. (2007) Prevalence, Awareness and Treatment of Cardiovascular Risk Factors in Patients at High Risk of Atherothrombosis in Japan. Circulation Journal, 71, 995-1003. https://doi.org/10.1253/circj.71.995

[8] Kumakura, H., Fujita, K., Kanai, H., Araki, Y., Hojo, Y., Kasama, S., et al. (2015) High-Sensitivity C-Reactive Protein, Lipoprotein(a) and Homocysteine Are Risk Factors for Coronary Artery Disease in Japanese Patients with Peripheral Arterial Disease. Journal of Atherosclerosis and Thrombosis, 22, 344-354. https://doi.org/10.5551/jat.25478

[9] Hirose, K., Chikamori, T., Hida, S., Tanaka, H., Igarashi, Y., Watanabe, Y., et al. (2009) Prevalence of Coronary Heart Disease in Patients with Aortic Aneurysm and/or Peripheral Artery Disease. American Journal of Cardiology, 103, 1215-1220. https://doi.org/10.1016/j.amjcard.2009.01.033

[10] Araki, Y., Kumakura, H., Kanai, H., Kasama, S., Sumino, H., Ichikawa, A., et al. (2012) Prevalence and Risk Factors for Cerebral Infarction and Carotid Artery Stenosis in Peripheral Arterial Disease. Atherosclerosis, 223, 473-477. https://doi.org/10.1016/j.atherosclerosis.2012.05.019

[11] Kumakura, H., Kanai, H., Matsuo, Y., Iwasaki, T. and Ichikawa, S. (2019) Asymptomatic Cerebral Infarction Is a Predictor of Long-Term Survival and Vascular or Limb Events in Peripheral Arterial Disease. European Heart Journal-Quality of Care and Clinical Outcomes, 5, 43-50. https://doi.org/10.1093/ehjqcco/qcy024

[12] Miura, T., Soga, Y., Doijiri, T., Aihara, H., Yokoi, H., Iwabuchi, M., et al. (2013) Prevalence and Clinical Outcome of Polyvascular Atherosclerotic Disease in Patients Undergoing Coronary Intervention. Circulation Journal, 77, 89-95.

https://doi.org/10.1253/circj.CJ-12-0535

[13] Anand, S.S., Caron, F., Eikelboom, J.W., Bosch, J., Dyal, L., Aboyans, V., et al. (2018) Major Adverse Limb Events and Mortality in Patients with Peripheral Artery Disease: The COMPASS Trial. Journal of the American College of Cardiology, 71, 2306 2315. https://doi.org/10.1016/j.jacc.2018.03.008

[14] Gerhard-Herman, M.D., Gornik, H.L., Barrett, C., et al. (2017) 2016 AHA/ACC Guideline on the Management of Patients with Lower Extremity Peripheral Artery Disease: Executive Summary: A Report of the American College of Cardiology/American Heart Association Task Force on Clinical Practice Guidelines. Circulation, 135, e686- 
e725. https://doi.org/10.1161/CIR.0000000000000470

[15] Yalim, Z., Aldemir, M. and Yalim, S.A. (2020) Assessment of the Relationship between Death and CHA2DS2-VASc Score in Peripheral Artery Disease. International Angiology, 39, 509-516. https://doi.org/10.23736/S0392-9590.20.04498-3

[16] Gutierrez, J.A., Scirica, B.M., Bonaca, M.P., et al. (2019) Prevalence and Outcomes of Polyvascular (Coronary, Peripheral, or Cerebrovascular) Disease in Patients with Diabetes Mellitus (from the SAVOR-TIMI 53 Trial). American Journal of Cardiology, 123, 145-152. https://doi.org/10.1016/j.amjcard.2018.09.014

[17] Verma, S., Mazer, C.D., Inzucchi, S.E., et al. (2021) Impact of Polyvascular Disease with and without Co-Existent Kidney Dysfunction on Cardiovascular Outcomes in Diabetes: A Post Hoc Analysis of EMPA-REG Outcome. Diabetes, Obesity \& Metabolism, 23, 1173-1181. https://doi.org/10.1111/dom.14326

[18] Expert Committee on the Diagnosis and Classification of Diabetes Mellitus (2003) Report of the Expert Committee on the Diagnosis and Classification of Diabetes Mellitus. Diabetes Care, 26, S5-S20. https://doi.org/10.2337/diacare.26.2007.S5

[19] Matsuo, S., Imai, E., Horio, M., Yasuda, Y., Tomita, K., Nitta, K., et al. (2009) Revised Equations for Estimated GFR from Serum Creatinine in Japan. American Journal of Kidney Diseases, 53, 982-992. https://doi.org/10.1053/j.ajkd.2008.12.034

[20] Kumakura, H., Kanai, H., Araki, Y., Hojo, Y., Iwasaki, T. and Ichikawa, S. (2015) 15-Year Patency and Life Expectancy After Primary Stenting Guided by Intravascular Ultrasound for Iliac Artery Lesions in Peripheral Arterial Disease. JACC: Cardiovascular Interventions, 8, 1893-1901. https://doi.org/10.1016/j.jcin.2015.08.020

[21] Thygesen, K., Alpert, J.S., Jaffe, A.S., Simoons, M.L., Chaitman, B.R., White, H.D., et al. (2012) Third Universal Definition of Myocardial Infarction. Circulation, 126, 2020-2035. https://doi.org/10.1161/CIR.0b013e31826e1058

[22] Ranke, C., Creutzig, A. and Alexander, K. (1992) Duplex Scanning of the Peripheral Arteries: Correlation of the Peak Velocity Ratio with Angiographic Diameter Reduction. Ultrasound in Medicine and Biology, 18, 433-440. https://doi.org/10.1016/0301-5629(92)90082-L

[23] Gutierrez, J.A., Mulder, H., Jones, W.S., Rockhold, F.W., Baumgartner, I., Berger, J.S., et al. (2018) Polyvascular Disease and Risk of Major Adverse Cardiovascular Events in Peripheral Artery Disease: A Secondary Analysis of the EUCLID Trial. JAMA Network Open, 1, Article ID: e185239. https://doi.org/10.1001/jamanetworkopen.2018.5239

[24] Gage, B.F., van Walraven, C., Pearce, L., Hart, R.G., Koudstaal, P.J., Boode, B.S., et al. (2004) Selecting Patients with Atrial Fibrillation for Anticoagulation: Stroke Risk Stratification in Patients Taking Aspirin. Circulation, 110, 2287-2292. https://doi.org/10.1161/01.CIR.0000145172.55640.93

[25] McQueen, M.J., Hawken, S., Wang, X., Ounpuu, S., Sniderman, A., Probstfield, J., et al. (2008) Lipids, Lipoproteins, and Apolipoproteins as Risk Markers of Myocardial Infarction in 52 Countries (The INTERHEART Study): A Case-Control Study. Lancet, 372, 224-233. https://doi.org/10.1016/S0140-6736(08)61076-4

[26] Kumakura, H., Kanai, H., Hojo, Y., Iwasaki, T. and Ichikawa, S. (2017) Long-Term Survival and Fate of the Leg in de Novo Intermittent Claudication. European Heart Journal-Quality of Care and Clinical Outcomes, 3, 208-215.

https://doi.org/10.1093/ehjqcco/qcw057

[27] Sarnak, M.J., Levey, A.S., Schoolwerth, A.C., Coresh, J., Culleton, B., Hamm, L.L., et al. (2003) Kidney Disease as a Risk factor for Development of Cardiovascular Disease: A Statement from the American Heart Association Councils on Kidney in Cardiovascular Disease, High Blood Pressure Research, Clinical Cardiology, and Epi- 
demiology and Prevention. Circulation, 108, 2154-2169. https://doi.org/10.1161/01.CIR.0000095676.90936.80

[28] Hojo, Y., Kumakura, H., Kanai, H., Iwasaki, T., Ichikawa, S. and Kurabayashi, M. (2016) Lipoprotein(a) Is a Risk Factor for Aortic and Mitral Valvular Stenosis in Peripheral Arterial Disease. European Heart Journal-Cardiovascular Imaging, 17, 492-497. https://doi.org/10.1093/ehjci/jev338

[29] Matsuo, Y., Kumakura, H., Kanai, H., Iwasaki, T. and Ichikawa, S. (2020) The Geriatric Nutritional Risk Index Predicts Long-Term Survival and Cardiovascular or Limb Events in Peripheral Arterial Disease. Journal of Atherosclerosis and Thrombosis, 27, 134-143. https://doi.org/10.5551/jat.49767

[30] Mehler, P.S., Coll, J.R., Estacio, R., Esler, A., Schrier, R.W. and Hiatt, W.R. (2003) Intensive Blood Pressure Control Reduces the Risk of Cardiovascular Events in Patients with Peripheral Arterial Disease and Type 2 Diabetes. Circulation, 107, 753-756. https://doi.org/10.1161/01.CIR.0000049640.46039.52

[31] Alkhalil, M., Kuzemczak, M., Whitehead, N., Kavvouras, C. and Dzavik, V. (2021) Meta-Analysis of Intensive Lipid-Lowering Therapy in Patients with Polyvascular Disease. Journal of the American Heart Association, 10, Article ID: e017948. https://doi.org/10.1161/JAHA.120.017948

[32] Drozda, J.P.J., Ferguson, T.B.J., Krumholz, H.M., Nallamothu, B.K., Olin, J.W., et al. (2016) 2015 ACC/AHA Focused Update of Secondary Prevention Lipid Performance Measures: A Report of the American College of Cardiology/American Heart Association Task Force on Performance Measures. Circulation: Cardiovascular Quality and Outcomes, 9, 68-95. https://doi.org/10.1161/HCQ.0000000000000014

[33] Antoniou, G.A., Fisher, R.K., Georgiadis, G.S., Antoniou, S.A. and Torella, F. (2014) Statin Therapy in Lower Limb Peripheral Arterial Disease: Systematic Review and Meta-Analysis. Vascular Pharmacology, 63, 79-87. https://doi.org/10.1016/j.vph.2014.09.001

[34] Ramos, R., Garcia-Gil, M., Comas-Cufi, M., Quesada, M., Marrugat, J., Elosua, R., et al. (2016) Statins for Prevention of Cardiovascular Events in a Low-Risk Population with Low Ankle Brachial Index. Journal of the American College of Cardiology, 67, 630-640. https://doi.org/10.1016/j.jacc.2015.11.052 